\title{
Therapeutic Ex Vivo-expanded Allogeneic gamma delta T-cells
}

National Cancer Institute

\section{Source}

National Cancer Institute. Therapeutic Ex Vivo-expanded Allogeneic gamma delta T-cells. NCI Thesaurus. Code C157487.

An off-the-shelf preparation of a subset of therapeutic, ex vivo-expanded, allogeneic Tlymphocytes that express only gamma chain and delta chain T-cell receptors (TCRs), with potential immunomodulating and antineoplastic activities. Upon administration of the therapeutic ex vivo-expanded allog eneic gamma delta T-cells, these cells secrete interferon-gamma (IFN-g) and exert direct killing of tumor cells. In addition, these cells activate the immune system to exert a cytotoxic T-lymphocyte $(C T L)$ response against tumor cells. Gamma delta T-lymphocytes play a key role in the activation of the immune system and do not require major histocompatibility complex (MHC)-mediated antigen presentation to exert their cytotoxic effect. 